\title{
Damped Ly $\alpha$ systems as probes of chemical evolution over cosmological timescales
}

\author{
Miroslava Dessauges-Zavadsky ${ }^{1}$ \\ ${ }^{1}$ Geneva Observatory, University of Geneva, 51, Ch. des Maillettes, 1290 Sauverny, Switzerland \\ email: miroslava.dessauges@unige.ch
}

\begin{abstract}
We review the current state of knowledge of damped Ly $\alpha$ systems (DLAs) selected in absorption on quasar sightlines. These objects are extremely useful to study the interstellar medium of high-redshift galaxies and the nucleosynthesis in the early Universe. The characteristics of this galaxy population has been investigated for years and slowly we are getting information on their puzzling nature. Imaging at $z<1$ shows that DLAs are associated with a mixing bag of galaxies with no especially large contribution from dwarf galaxies. Evidence for a mild evolution of the cosmic mean metallicity with time is observed. The star formation histories of these high-redshift galaxies begin to be accessible and indicate that DLAs tend to be young, gas-dominated galaxies with low star formation rates per unit area. Finally, indirect estimation of the DLA stellar masses from the mass-metallicity relations observed for emission-selected star-forming galaxies at $z=2-3$ points to intermediate-mass galaxies with $M_{*}<10^{9} \mathrm{M}_{\odot}$.
\end{abstract}

Keywords. ISM: abundances, galaxies: evolution, galaxies: high-redshift, quasars: absorption lines

\section{Introduction}

Prior to the advent of the 8-10 m class telescopes, the knowledge of galaxies at $z>0.5$ relied almost exclusively on galaxies detected in absorption, and in particular on the so-called damped Ly $\alpha$ systems (DLAs), detected in QSO sightlines with the highest H I column densities $\left(N(\mathrm{HI})>2 \times 10^{20} \mathrm{~cm}^{-2}\right)$. The first dedicated large survey for DLAs was done by Wolfe et al. (1986), and nowadays more than 1000 DLAs have been identified in the Sloan Digital Sky Survey (SDSS, Prochaska et al. 2005†). The study of DLAs is extremely valuable, since DLAs contain most of the neutral hydrogen available for star formation in the Universe, arise in the interstellar medium (ISM) of protogalaxies, and are believed to be the progenitors of present-day normal galaxies. Moreover, the selection of these high-redshift galaxies solely on their H I column density presents several advantages compared to the selection techniques in emission (see Wolfe et al. 2005 for a review): (i) it is independent from the galaxy's brightness and leads to a large diversity of luminosities and masses; (ii) it provides a better sampling of the global star formation history of the Universe; (iii) it allows to characterize the physical properties of the ISM of high-redshift galaxies; and (iv) it gives access to comprehensive samples of elemental abundances that give constraints on the galactic chemical evolution. The major drawback is the single line of sight at disposal to characterize the global properties of the high-redshift galaxy.

What have we learnt so far on this galaxy population? Various DLA studies - imaging, neutral gas mass density census, analysis of chemical properties, kinematics - have been undertaken with the common goal that aimed at understanding the nature of this galaxy population and its link with other high-redshift galaxies identified with other selection techniques. However, despite decades of studies, it is still not clear whether the observed

$\dagger$ http://www.ucolick.org/ xavier/SDSSDLA/index.html 
cold neutral gas associated with DLAs traces the ISM of spiral galaxies, of halos of galaxies, or of dwarf galaxies. In what follows, we will try to review the different results obtained so far.

\section{DLA imaging}

DLA imaging is extremely challenging, because of the strong glare of the background QSO which makes it very difficult to detect a foreground faint galaxy located only a few arcsec away from the QSO sightline. However, imaging provides crucial information in the understanding of the DLA nature by constraining the morphological type, the star formation rate, and the impact parameter which probes the extension of the neutral gas around these high-redshift galaxies.

At $z<1$, the identification of the DLA counterparts has been successful for 13 systems so far (e.g. Le Brun et al. 1997; Chen \& Lanzetta 2003; Rao et al. 2003; Chen et al. 2005). It appears that DLAs are associated with a mixing bag of galaxies, $45 \%$ being disk dominated, $22 \%$ bulge dominated, $11 \%$ irregulars, and $22 \%$ being located in galaxy groups. As a consequence, DLA galaxies at $z<1$ seem to be representative of the field galaxy population, with an especially large contribution from dwarf galaxies being not necessary. Their average luminosity, $L_{\mathrm{B}} \simeq 0.5 L_{\mathrm{B}}^{*}$, points to a sub- $L^{*}$ galaxy population and the impact parameters reach $r=24-30 h^{-1} \mathrm{kpc}$.

At $z>1$, the identification of the DLA counterparts is even more challenging and so far only 7 DLA galaxies have been detected, among them 3 with $z_{\mathrm{DLA}} \approx z_{\mathrm{QSO}}$ (e.g. Warren et al. 2001; Møller et al. 2002; Møller et al. 04; Weatherley et al. 2005). The observed Ly $\alpha$ luminosity varies from 1 to $10 \times 10^{42} \mathrm{erg} \mathrm{s}^{-1}$, corresponding to $L_{\mathrm{B}} \simeq 0.6 L_{\mathrm{B}}^{*}$. This leads to a star formation rate (SFR) of 10 to $30 \mathrm{M}_{\odot} \mathrm{yr}^{-1}$. Møller et al. (2004) suggested the existence of a possible luminosity-metallicity relation with the detected high-redshift DLA galaxies showing higher metallicities than the undetected ones.

\section{Census of the neutral gas mass density with DLAs}

The neutral gas mass density is defined as:

$$
\Omega_{g}=\frac{\mu m_{\mathrm{H}} H_{0}}{c \rho_{c}} \frac{\Sigma N(\mathrm{HI})}{\Delta X}
$$

where $\mu$ is the mean molecular mass of the gas, $H_{0}$ the Hubble constant, $\rho_{c}$ the critical mass density, and $\Delta X$ the total path length. $\Omega_{g}$ has an important cosmological significance. Its evolution with cosmic time constrains the build-up of structure within hierarchical cosmology and represents a key ingredient in models of galaxy formation. It serves as the neutral gas reservoir for star formation at high redshift and describes the competition between gas accretion and star formation.

The first DLA surveys have reported statistical error on $\Omega_{g}$ of about $30 \%$ in redshift intervals of $\Delta z \approx 0.5$ at high redshift (e.g. Storrie-Lombardi \& Wolfe 2000). With the SDSS, Prochaska et al. (2005) managed to derive $\Omega_{g}$ with a precision better than $10 \%$ (see Fig. 1). They found the first evidence for significant evolution with a $50 \%$ decrease of $\Omega_{g}$ from $z=3.3$ to $z=2.3$. It is, however, still not clear how this decrease can be explained - with star formation it seems unlikely, since the SFR peaks below $z=2$, and with ionization it seems unlikely as well, since the intensity of the extragalactic background radiation field is constant - and how it can be reconciled with a flat gas mass density from $z=2$ to today as supported by the low-redshift DLA survey made by Rao et al. (2006). 


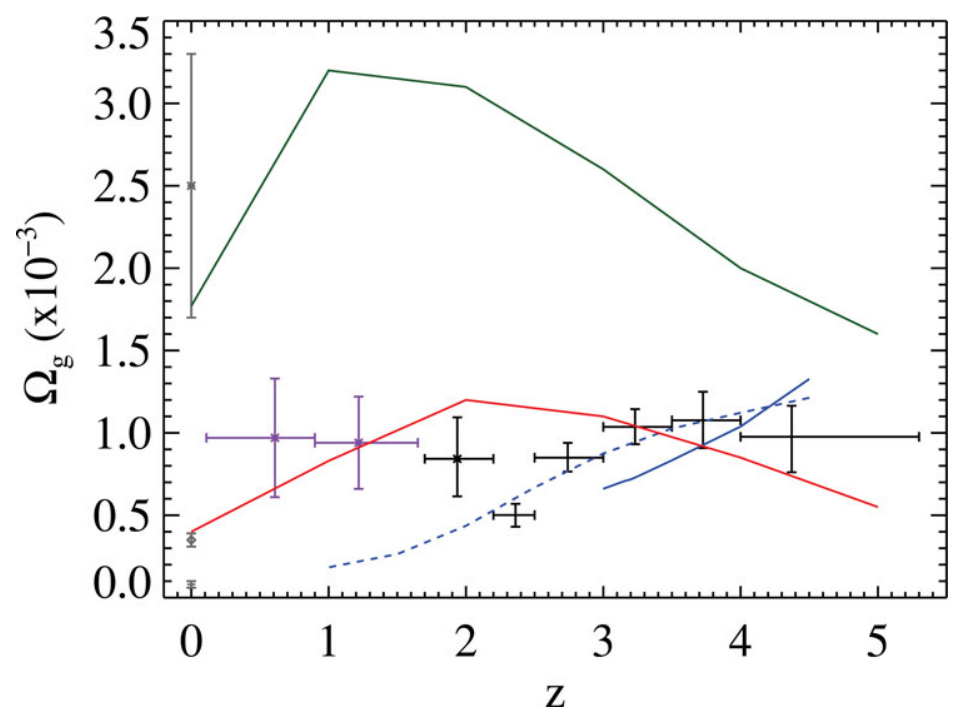

Figure 1. DLA neutral gas mass density obtained at $z>1.5$ from the SDSS by Prochaska et al. (2005) and at $z<1.5$ by Rao et al. (2006). These observations are compared to different theoretical models. The data points at $z=0$ correspond from top to bottom to the stellar mass density, the neutral gas mass density of local galaxies, and the mass density of dwarf irregular galaxies. Figure reproduced from Prochaska et al. (2005).

The comparison with the stellar mass density shows that it is 3 times higher than the DLA gas mass density at $z \sim 3$ and suggests that every galaxy is (or was) a DLA. On the other hand, the local gas mass density matches the one of DLAs at $z \sim 2$ and indicates that the SFR seems to balance the accretion. Finally, the dwarf mass density appears to be 10 times smaller than the DLA gas mass density, showing that the majority of DLAs cannot evolve into dwarf galaxies.

\section{DLA chemical properties}

\subsection{DLA metallicity evolution}

Evidence for metallicity evolution as traced by DLAs has been investigated by several groups (e.g. Pettini et al. 1999; Vladilo et al. 2000; Prochaska \& Wolfe 2000; Prochaska et al. 2003; Kulkarni \& Fall 2002; Kulkarni et al. 2005). Currently, metallicity measurements of 162 DLAs are available from moderate- to high-resolution spectroscopy. They are assembled in Fig. 2 and show the following characteristics: (i) a large scatter of about 1 dex that is roughly constant with redshift and that can be explained by a combination of gas cross-section selection, metallicity gradients and/or different star formation histories; (ii) very few DLAs with solar metallicities in contrast with emission-selected galaxies like the Lyman-break galaxies (LBGs, e.g. Erb et al. 2006a); and (iii) a metallicity floor at $[\mathrm{M} / \mathrm{H}] \sim-3$, a value that yet significantly exceeds the detection limit of the current instruments. This questions the existence of primordial gas in the ISM of high-redshift galaxies, but on the other hand supports the idea that DLAs are linked to current/recent star formation that takes place within these galaxies.

The data show a mild evolution with redshift in both the unweighted mean metallicities (Fig. 2, large red crosses) and in the weighted mean metallicities by the H I column densities (Fig. 2, large black crosses). A decrease by -0.25 dex per redshift-bin is observed, which corresponds to a metallicity decrease by a factor of 2 per Gyr. A key implication 


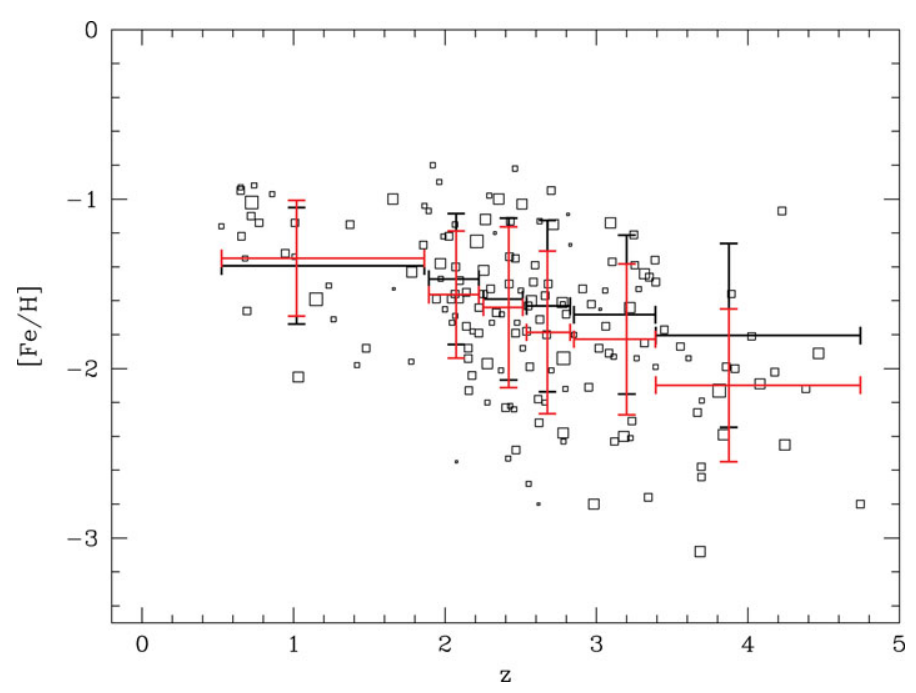

Figure 2. $[\mathrm{Fe} / \mathrm{H}]$ metallicity plotted as a function of redshift for the 162 DLA measurements currently available (open squares with size proportional to the measurement errors). The large black crosses correspond to the $\mathrm{H}$ I weighted mean metallicities and the large red crosses to the unweighted mean metallicities. Both highlight a mild metallicity evolution with redshift.

of this result comes to light when comparing the average DLA metallicity at $z=2.5$ with the integral under the DLA cosmic star formation history at $z>2.5$ (Wolfe et al. 2003. The integrated star formation rate implies $3-10$ times the mass density of metals observed in DLAs, highlighting the so-called "missing metals problem", first pointed out by Pettini (1999). This suggests several possible implications: (i) DLAs may not be representative of the global galaxy population; (ii) DLAs may be dominated by dwarf or low surface brightness galaxies, as supported by the cross-section selection effect which tends to reveal faint galaxies that have larger cross-sections than bright galaxies, but is in contradiction with the $\mathrm{H}$ I studies at $z \sim 0$ showing that most $\mathrm{H}$ I resides in large spirals and with DLA imaging at $z<1$; and (iii) the DLA selection may be biased against metal-rich systems.

Pei et al. (1991) was the first to suggest that DLAs with the highest metal column densities may be those with the highest dust content and may obscure the background QSO. This led Ellison et al. to build up a survey of DLAs in front of radio-loud QSOs which selection should be free from the dust obscuration effect. They showed that radioloud QSOs point to a mild reddening and to small differences with optically-selected QSOs, both in the DLA neutral gas mass density, the number density of strong Mg II systems and the DLA average metallicity (Ellison et al. 2001; Ellison et al. 2004; Akerman et al. 2005). These results were further confirmed by Murphy \& Liske (2004) who reviewed all the SDSS QSOs with and without DLAs and found that the dust-reddening caused by foreground DLAs is small at $z \sim 3$ with $E(\mathrm{~B}-\mathrm{V})<0.02 \mathrm{mag}$.

\subsection{DLA abundance measurement uncertainties}

There are two main effects that harm elemental gas-phase abundance measurements: dust and ionization. Indeed, a fraction of elements may not be detected in gas-phase, if locked in dust grains and/or if ionized. Dust depletion effects have been highlighted in DLAs when looking at the abundance ratios of two elements with the same nucleosynthetic origins - Fe-peak elements or $\alpha$-elements. They tend to resemble the dust depletion effects observed in the Galactic warm halo gas (e.g. Hou et al. 2001). There are three possible 
ways to tackle the problem of dust depletion: (i) the study of dust-free DLAs only with $[\mathrm{Zn} / \mathrm{Fe}]<0.3$ dex; (ii) the analysis of mildly refractory elements solely (N, O, S, Ar, and $\mathrm{Zn}$ ); and (iii) the computation of dust depletion corrections (prescriptions of e.g. Vladilo 2002).

The high H I column densities observed in DLAs mostly imply that the bulk of the neutral gas is self-shielded. The dominant ionization states hence are the neutral one for elements with the first ionization potential $>13.6 \mathrm{eV}\left(\mathrm{O}^{0}, \mathrm{~N}^{0}\right)$ and the singly ionized one for elements with the first ionization potential $<13.6 \mathrm{eV}\left(\mathrm{Si}^{+}, \mathrm{Fe}^{+}\right)$. The ionization effects are generally estimated as unimportant with an ionization fraction $x=\mathrm{H}^{+} /\left(\mathrm{H}^{0}+\right.$ $\left.\mathrm{H}^{+}\right)<10$ and ionization corrections lower than abundance measurement errors (e.g. Viegas 1995; Howk \& Sembach 1999; Vladilo et al. 2001). However, > $10 \%$ of DLAs are certainly significantly ionized (Prochaska et al. 2002). Consequently, to derive intrinsic abundance measurements of a DLA, dust depletion and ionization effects have to be carefully evaluated.

\subsection{DLA abundance patterns}

In high-resolution and good signal-to-noise ratio spectra $(\mathrm{S} / \mathrm{N}>20$ per pixel) obtained on a 8-10 m class telescope, column densities of more than 50 ions and abundances of 16 elements are accessible in DLAs: $5 \alpha$-elements $\mathrm{O}, \mathrm{Mg}, \mathrm{Si}, \mathrm{S}, \mathrm{Ti} ; 6$ Fe-peak elements $\mathrm{Cr}$, Mn, Fe, Co, Ni, Zn; and 5 other elements C, N, Al, P, Ar (see Dessauges-Zavadsky et al. 2006). Is this information sufficient to constrain the star formation histories (SFHs) of these high-redshift galaxies?

On the theoretical point of view, the information at disposal is sufficient, since the $\mathrm{SFH}$ is entirely determined by the relative abundances versus absolute abundances and the age is constrained by the relative abundances versus the redshift. Indeed, the relative abundances depend on the stellar lifetimes, the yields and the initial mass function and hence play the role of cosmic clocks when considering two elements produced on different timescales $([\alpha /$ Fe-peak $],[\mathrm{N} / \alpha])$, while the absolute abundances directly depend on the SFH type. This implies that a weak star formation or a strong+fast star formation will yield to different abundance patterns (see the contribution by Francesca Matteucci).

In practice, things are not so straightforward, mainly because of dust and ionization effects that may mimic nucleosynthetic effects. There is, for instance, an old controversy on the real $\alpha$-enhancement in DLAs, usually traced by the [Si/Fe] ratio known to be contaminated by dust depletion effects (Prochaska \& Wolfe 2002; Dessauges-Zavadsky et al. 2006). Systems with low dust contents selected with $[\mathrm{Zn} / \mathrm{Fe}]<0.3$ dex show [Si $/ \mathrm{Fe}]$ ratios between 0.15 to 0.35 dex, while systems with $[\mathrm{Zn} / \mathrm{Fe}]>0.3$ dex show $[\mathrm{Si} / \mathrm{Fe}]$ ratios reaching up to $0.8-0.9$ dex. However, the large number of elements and ions accessible in the available QSO spectra now allow to accurately apprehend the dust depletion and ionization effects, when DLAs are studied individually.

\subsection{DLA star formation histories}

To determine the SFHs and ages of the DLA galaxies, a grid of chemical evolution models has first to be built up. The models are then fitted to reproduce the observed DLA abundance patterns. Two types of chemical evolution models identified as the "spiral models" (Chiappini et al. 1997) and the "dwarf irregular models" (Bradamante et al. 1998), according to the type of galaxies they do match best, are usually considered for DLAs. The spiral models are constructed on the basis of the inside-out scenario for the disk formation with the star formation proceeding faster in the inner regions than in the outer regions. This implies that different abundance patterns are expected at different galactocentric distances. In dwarf irregular models, star formation may proceed 

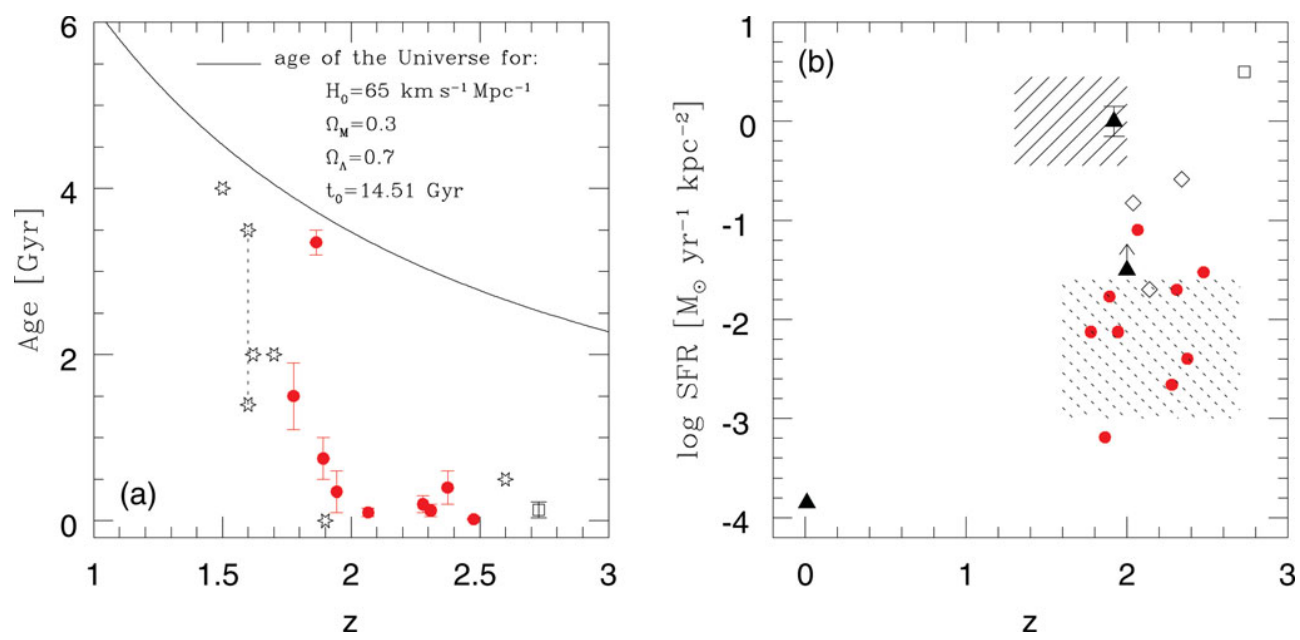

Figure 3. Left. Age distribution as a function of redshift for DLA galaxies (filled circles), emission-selected galaxies (stars), and the LBG MS 1512-cB58 (square). Right. Star formation rate per unit area distribution as a function of redshift. The DLAs are shown by filled circles. The dotted-shaded area corresponds to the SFRs obtained for DLAs by Wolfe et al. (2003) from the $\mathrm{C}$ II fine-structure line and the dashed-shaded area corresponds to the SFRs obtained for the emission-selected galaxies. The triangles represent the three DLAs for which we have an estimation of their SFR from emission lines, the square corresponds to the LBG MS 1512-cB58, and the diamonds to the gamma-ray burst host galaxies.

either in bursts separated by quiescent periods or at a low regime but continuously. This implies the need to constrain three free parameters: the star formation efficiency, the burst duration, and the time of occurrence of the burst.

This exercise has been successfully applied to 9 DLAs so far (Dessauges-Zavadsky et al. 2004; Dessauges-Zavadsky et al. 2007). No single SFH explains the diverse sets of DLA abundance patterns: 6/9 DLAs have SFHs typical of dwarf irregular bursting star formation, 1/9 DLA has a SFH typical of dwarf irregular continuous star formation (continuous SFH), and only 2/9 DLAs (each at $z<2$ ) have SFHs typical of spiral outer disks (episodic bursting SFH). The common characteristic is that DLAs are weak star-forming galaxies, models with high star formation efficiencies are ruled out. The derived DLA star formation rates per unit area are moderate or low, with values between $-3.2<\log \mathrm{SFR}<-1.1 \mathrm{M}_{\odot} \mathrm{yr}^{-1} \mathrm{kpc}^{-2}$ (see Fig. 3, right panel). They are in good agreement with the DLA SFRs obtained from the fine-structure C II line by Wolfe et al. (2003), however they are $10-1000$ times lower than the SFRs of the emission-selected galaxies at the same redshifts (Savaglio et al. 2004; Erb et al. 2006b). DLAs are dominated by young objects with ages between $20-600 \mathrm{Myr}$ (see Fig. 3, left panel). One DLA at $z_{\text {abs }}=1.864$ in our sample has an age estimated to more than $3 \mathrm{Gyr}$, suggesting that galaxies were already formed at $z>10$.

\section{DLA kinematics}

In QSO high-resolution spectra obtained on a 8-10 m class telescope, the metal-line profiles are resolved into clouds that trace the velocity fields of the DLA high-redshift galaxies. The reconstruction of the nature of these velocity fields - rotation, outflows, turbulence - is, however, very challenging with individual one-dimensional sightlines. The velocity widths, $\Delta V$, are measured from unsaturated low-ionization lines and are often defined as the interval that contains $90 \%$ of the integrated optical width. 
The large data sample currently available allowed recently to confirm the existence of a velocity-metallicity correlation for DLA galaxies (Ledoux et al. 2006; Prochaska et al. 2008). This correlation is shown in Fig. 2 from Ledoux et al. (2006) and the best-fit linear relation yields: $[\mathrm{X} / \mathrm{H}]=1.55( \pm 0.12) \log \Delta V-4.33( \pm 0.23)$. The observed dispersion, larger than the metallicity measurement uncertainties, can be explained by (i) different sightline impact parameters, (ii) different galaxy inclinations, and (iii) radial gradients in metallicity. The data also support the evidence for a redshift evolution of the velocitymetallicity relation with the median metallicity and median velocity width increasing with decreasing redshift: $[\mathrm{X} / \mathrm{H}]=-1.59, \Delta V=69 \mathrm{~km} \mathrm{~s}^{-1}$ at $z>2.4$ and $[\mathrm{X} / \mathrm{H}]=-1.15$, $\Delta V=92 \mathrm{~km} \mathrm{~s}^{-1}$ at $z<2.4$.

It is very tempting to interpret this DLA velocity-metallicity correlation, observed over more than a factor of 100 spread in metallicity, as the consequence of an underlying mass-metallicity relation for the galaxies associated with the DLA absorption. Several arguments favor this interpretation. First, peculiar ejection of hot gas should primarily affect the kinematics of high-ionization lines such as C IV and Si IV, the measurements of velocity widths based on low-ionization lines should instead be dominated by motions on galactic scale governed, or induced, by gravity. Second, Prochaska \& Wolfe (1997) proposed that the observed asymmetries in the line profiles may be explained by rotating large disks. Third, the observed line profile velocity broadening may also result from the merging of proto-galactic clumps, in this case $\Delta V$ is also connected to gravity, being a good indicator of the circular velocity of the underlying dark-matter halo (Haehnelt et al. 1998). Fourth, as pointed out in Sect. 2, DLAs successfully imaged at $z>2$ have high metallicities, suggesting that stellar mass correlates with enrichment. There are two counterarguments to interpreting the measured velocity widths as the tracers of the gravitational potential. First, it may be unrealistic to explain $\Delta V>200 \mathrm{~km} \mathrm{~s}^{-1}$ in terms of a single galactic potential, additional velocity fields certainly contribute. These could include galactic-scale outflows, but also peculiar motions of multiple galaxies along the sightline (e.g. Maller et al. 2001). Second, Bouché et al. (2006) reported an anticorrelation between $\mathrm{Mg}$ II equivalent width and the cluster length of these absorbers with large red galaxies that they interpreted as the result of a wind scenario: systems exhibiting larger equivalent widths occur in less massive, star-bursting galaxies.

On balance, the interpretation of the velocity-metallicity relation as the mass-metallicity relation is favored, moreover that a mass-metallicity relation for local galaxies is well established (e.g. Tremonti et al. 2004). A mass-metallicity relation is also observed for galaxies at $0.4<z<1$ from the Gemini Deep Deep Survey and the Canada-France Redshift Survey (Savaglio et al. 2005) and for UV-selected galaxies at $z \sim 2.2$ and at $z \sim 3.5$ (Erb et al. 2006a and Maiolino et al. 2008, respectively). For discussion on the evolution of the mass-metallicity relation with redshift, we refer the readers to the contribution by Filippo Mannucci. We would like just to point out that the observed redshift evolution of the DLA velocity-metallicity relation is consistent with the evolution of the mass-metallicity relation. Assuming DLAs at $z=2-3$ follow a similar mass-metallicity relation as the high-redshift UV-selected galaxies, we can infer an upper limit to their stellar masses of $10^{9} \mathrm{M}_{\odot}$. This suggests that DLAs are intermediate-mass galaxies.

\section{References}

Akerman, C. J., Ellison, S. L., Pettini, M., \& Steidel, C. C. 2005, A $\& A, 440,499$

Bouché, N., Murphy, M. T., Péroux, C., Csabai, I., \& Wild, V. 2006, MNRAS, 371, 495

Bradamante, F., Matteucci, F., \& D'Ercole, A. 1998, A\& A, 337, 338

Chen, H.-W. \& Lanzetta, K. M. 2003, ApJ, 597, 706 
Chen, H.-W., Kennicutt, R. C. Jr., \& Rauch, M. 2005, ApJ, 620, 703

Chiappini, C., Matteucci, F., \& Gratton, R. 1997, ApJ, 477, 765

Dessauges-Zavadsky, M., Calura, F., Prochaska, J. X., D’Odorico, S., \& Matteucci, F. 2004, $A \mathscr{E} A, 416,79$

Dessauges-Zavadsky, M., Prochaska, J. X., D'Odorico, S., Calura, F., \& Matteucci, F. 2006, $A \mathscr{E} A, 445,93$

Dessauges-Zavadsky, M., Calura, F., Prochaska, J. X., D’Odorico, S., \& Matteucci, F. 2007, $A \& A, 470,431$

Ellison, S. L., Yan, L., Hook, I. M., Pettini, M., Wall, J. V., \& Shaver, P. 2001, A\& A, 379, 393

Ellison, S. L., Churchill, C. W., Rix, S., \& Pettini, M. 2004, ApJ, 615, 118

Erb, D. K., Shapley, A. E., Pettini, M., Steidel, C. C., Reddy, N. A., \& Adelberger, K.L. 2006, ApJ, 644, 813

Erb, D. K., Steidel, C. C., Shapley, A. E., Pettini, M., Reddy, N. A., \& Adelberger, K. L. 2006, ApJ, 647, 128

Haehnelt, M. G., Steinmetz, M., \& Rauch, M. 1998, ApJ, 495, 647

Hou, J. L., Boissier, S., \& Prantzos, N. 2001, A\&A, 370, 23

Howk, J. C. \& Sembach, K. R. 1999, ApJ, 523, L141

Kulkarni, V. P. \& Fall, S. M. 2002, 580, 732

Kulkarni, V. P., Fall, S. M., Lauroesch, J. T., York, D. G., Welty, D. E., Khare, P., \& Truran, J. W. 2005, ApJ, 618, 68

Le Brun, V., Bergeron, J., Boissé, P., \& Deharveng, J. M. 1997, A\& 4 A, 321, 733

Ledoux, C., Petitjean, P., Fynbo, J. P., Møller, P., \& Srianand, R. 2006, A\&A, 457, 71

Maiolino, R., et al., 2008, A\&BA, submitted [arXiv:0806.2410]

Maller, A. H., Prochaska, J. X., Somerville, R. S., \& Primack, J. R. 2001, MNRAS, 326, 1475

Møller, P., Warren, S. J., Fall, S. M., Fynbo, J. U., \& Jakobsen, P. 2002, ApJ, 574, 51

Møller, P., Fynbo, J. P. U., \& Fall, S. M. 2004, A\& A, 422, L33

Murphy, M. T. \& Liske, J. 2004, MNRAS, 354, L31

Pei, Y. C., Fall, S. M., \& Bechtold, J. 1991, ApJ, 378, 6

Pettini, M., Ellison, S. L., Steidel, C. C., \& Bowen, D. V. 1999, ApJ, 510, 576

Pettini, M. 1999, in: J. R. Walsch, M. R. Rosa (eds), ESO Workshop, Springer-Verlag, p. 233

Prochaska, J. X. \& Wolfe, A. M. 2000, ApJ, 533, L5

Prochaska, J. X., Henry, R. B. C., O'Meara, J. M., Tytler, D., Wolfe, A. M., Kirkman, D., Lubin, D., \& Suzuki, N. 2002, PASP, 114, 933

Prochaska, J. X. \& Wolfe, A. M. 2002, ApJ, 566, 68

Prochaska, J. X., Gawiser, E., Wolfe, A. M., Castro, S., \& Djorgovski, S. G. 2003, ApJ, 595, L9

Prochaska, J. X., Herbert-Fort, S., \& Wolfe, A. M. 2005, ApJ, 635, 123

Prochaska, J. X., Chen, H.-W., Wolfe, A. M., Dessauges-Zavadsky, M., \& Bloom, J. S. 2008, ApJ, 672, 59

Rao, S. M., Nestor, D. B., Turnshek, D. A., Lane, W. M., Mornier, E. M., \& Bergeron, J. 2003, ApJ, 595, 94

Rao, S. M., Turnshek, D. A., \& Nestor, D. B. 2006, 636, 610

Savaglio, S., et al., 2004, ApJ, 602, 51

Savaglio, S., et al., 2005, ApJ, 635, 260

Storrie-Lombardi, L. J. \& Wolfe, A. M. 2000, ApJ, 543, 552

Tremonti, C. A., et al. 2004, ApJ, 613, 898

Viegas, S. M. 1995, MNRAS, 276, 268

Vladilo, G., Bonifacio, P., Centurión, M., \& Molaro, P. 2000, ApJ, 543, 24

Vladilo, G., Centurión, M., Bonifacio, P., \& Howk, J. C. 2001, ApJ, 557, 1007

Vladilo, G. 2002, A\&A, 391, 407

Warren, S. J., Møller, P., Fall, S. M., \& Jakobsen, P. 2001, MNRAS, 326, 759

Weatherley, S. J., Warren, S. J., Møller, P., Fall, S. M., Fynbo, J. U., \& Croom, S. M. 2005, MNRAS, 358, 985

Wolfe, A. M., Turnshek, D. A., Smith, H. E., \& Cohen, R. D. 1986, ApJS, 61, 249

Wolfe, A. M., Gawiser, E., \& Prochaska, J. X. 2003, ApJ, 593, 235

Wolfe, A. M., Gawiser, E., \& Prochaska, J. X. 2003, ARA 8 A, 43, 861 